\title{
Study on the Coordination Mechanism of Creative Intellectual Resource Allocation Behavior in the Internet Copyright Industry from the Perspective of Evolutionary Game
}

\author{
Hui Cai ${ }^{1, a}$ \\ ${ }^{1}$ Business School, University of Shanghai for Science and Technology, Yangpu District, Shanghai, China \\ acaihui@usst.edu.cn
}

\begin{abstract}
The copyright value of cultural industry is developing rapidly through the Internet. The starting point of the value chain of the Internet copyright industry is the literary works with storyline on the Internet platform, the network literature works as meta-IP, with the support of Internet copyright developers, continue to be developed to form a series of cultural derivatives such as film and television, animation, online games. Internet copyright industry has become an important part of cultural and creative industries. Internet copyright developers and meta-IP creators in the process of allocation of creative intelligence resources have obvious evolutionary game characteristics, in the whole game link, meta-IP creators and copyright developers of the game is limited, Internet copyright developers evaluate and rearrange optimal development strategies based on the decision-making results of the meta-IP creators group. This study analyzes the dynamic evolution process of the system, discusses the different possible values of the influence factors, simulates the process of the system to achieve evolutionary equilibrium, and obtains more intuitive conclusions.
\end{abstract}

\section{演化博亦视角下的互联网版权产业创意智力资源配置 行为协调机制研究}

蔡茾 $^{1, a}$

${ }^{1}$ 上海理工大学管理学院, 杨浦区, 上海, 中国
${ }^{a}$ caihui@usst.edu.cn

摘要:

文化产业的版权价值通过互联网正急速发展，互联网版权产业价值链的起点是互联网平台上具有故事情节的文 学作品, 网络文学作品作为元 IP, 在互联网版权开发商的加持下, 持续得到开发形成影视、动漫、网游等一 系列文化衍生品。互联网版权产业已经成为文化创意产业的重要组成部分。互联网版权开发商和元 IP 创作者 在创意智力资源配置过程中具有明显的演化博弯特征, 在整个博亦环节, 元 IP 创作者与版权开发商的博弯是 有限次的, 互联网版权开发商依据元 IP 创作者群体的决策结果评估并重新安排最优发展战略。本研究对系统 的动态演化过程进行了分析, 通过对影响因子的不同可能取值进行了讨论, 对系统达到演化均衡的过程进行了 仿真, 从而得到较直观的结论。

关键词: 文化产业 互联网版权产业 演化博弯 版权开发 


\section{1 前言}

文化创意产业的版权价值创造与内容分发, 凭借 互联网信息传递的便捷高效、供需对接结构的扁平化、 内容呈现方式的矩阵化等属性, 在媒介市场中脱颖而 出。从版权产业这些属性可以看到, 互联网平台带给 版权产业的这些独特属性并不能有效降低版权开发 过程中参与主体间的信息不对称，IP 的原创作者与 版权开发机构之间存在一定的博亦关系。一方面, 版 权资源开发的成功不仅需要有一定数量的优秀网络 文学作家, 而且还需要一定数量的互联网版权开发商 参与, 而互联网版权开发商并不能准确评估出元 IP 创作者的行为选择动机以及最终的行为选择结果, 只 能猜测对方的决策进而形成已方的行为选择; 另一方 面, 元 IP 创作者可以根据后续的合作模式及收益情 况对自身的行为选择做出判断。互联网版权产业链的 发展和不断走向成熟的过程中, 促使版权具备了与众 不同的经验和特征。互联网版权产业存在长期的竞合 关系, 主要是行为主体之间在约束和依附基础上形成 的 ${ }^{[1]}$ 。鉴于此, 本研究将互联网版权开发商与元 IP 创作者之间的合作博弯作为切入点来研究, 确定博亦 参与方的学习机制和动态策略演化的过程, 对版权开 发过程中两方的博弃行为进行分析。

\section{2. 基于演化博弯视角的互联网版权产业智力 资源配置行为分析}

互联网版权开发商与元 IP 创作者之间存在与供 应链研究相类似的多阶段博峦关系 ${ }^{[2][3]}$ 。在互联网版 权产业创意智力资源配置的初始环节, 当 IP 开发成 功的概率达到自我预期时, 元 IP 创作者才会形成共 同合作开发意愿。在这个过程中主要借鉴以往信息。 若元 IP 创作者在互联网版权开发商上合作版权开发 失败, 则元 IP 创作者一般不会再在平台上重复进行 行为选择, 这时的博峦信息是不完全的 ${ }^{[4]}$ 。当项目 开始运行后, 互联网版权开发商针对前期对元 IP 创 作者的考察, 以及成本来选择是否对元 IP 创作者来 评估, 元 IP 创作者因缺失开发商的版权开发成本信 息从而难以确定其开发决策, 这次博弯基于单次不完 全信息。近年来, 学界以互联网版权开发商与元 IP 创作者之间的合作博弯研究数量有限 ${ }^{[5]}[6]$ 。

本研究从互联网版权智力资源配置合作行为的 协调机制出发, 结合利益分配以及行为选择的奖惩机 制等因素, 应用演化博亦构建双种群博亦模型, 重点 对产业价值链中涉及的各元素主体进行演进规律的 探讨。

\section{1 演化博絃论的适用性分析}

如上文所述, 在进行博弯关系分析时一定要考虑 信息的不对称 ${ }^{[7]}$ 。根据版权开发的实际需要, 如果互 联网版权开发商与元 IP 的开发者开展相关合作, 推
进版权开发进程, 互联网版权产业创意智力资源如果 能够合理有效的进行配置, 后续价值链上诸如游戏、 影视、文创产品等衍生行业就会创造更高的市场价值, 元 IP 创作者会获得对应的版权分账, 否则其仅能获 得元 IP 初始状态对应的收益（如网络文学内容向在 线阅读人群收费所得收益) ${ }^{[8]}$ 。影响元 IP 创作者在 后续的版权开发过程中获得的收益, 这种收益会对行 为主体形成反馈机制, 从而影响后一阶段参与主体的 行为选择。元 IP 创作者和互联网版权开发商对最终 收益的判断一般在版权开发效果的基础之上, 各参与 主体由于缺失对方信息, 其决策只能是基于“有限理 性”的, 这时经典博弯论的局限性就显示出来了 ${ }^{[9]}$ 。

从互联网版权开发的动态过程中可以看到, 版权 开发商和元 IP 创作者之间具有显著的演化博亦特征 指向, 他们两者之间在版权价值开发的博亦决策行为 在版权产业创意智力资源配置中非常重要: 都追求利 润最大化, 并需要在创意智力资源配置活动的初始阶 段根据上一阶段的行为结果做出选择。本研究从互联 网版权开发商与元 IP 创作者之间的合作博亦的角度, 对版权开发过程中两方的博弯行为进行分析, 重点通 过学习机制描述决策过程。

\section{2 互联网版权产业智力资源配置合作行为协 调的内容与影响因素}

互联网版权智力资源配置合作行为协调主要指 的是元 IP 创造者、版权营运平台之间朝着共同目标 而努力, 协商发展两方的行为, 合理分配合作总收益, 大家推动互联网版权产业价值链有序化演进的过程。 互联网版权智力资源配置合作行为协调包括价值链、 链接点等方面的协调:

（1）互联网版权产业价值链层面上的协调，是 指元 IP 创造者、版权营运平台之间合作行为的相互 协调。这一层面的有效协调能够降低成本, 提高整个 互联网版权产业价值链的管理水平和运作效率, 从而 提高整体绩效。

（2）链接节点层面上的协调, 是指元 IP 创造者、 版权营运平台各自内部行为的协调。元 IP 创造者内 部行为协调能够保证内容, 赋能 IP; 互联网版权产 业内部行为协调将重点保障实现新 IP 培育; 版权营 运平台内部行为协调则旨在完成新 IP 开发与市场对 接。

（3）创意智力资源是互联网版权产业开发的核 心要素资源, 也是版权资源开发过程以及成品质量、 生命力的决定性要素。追求收益最大化并对不确定性 进行控制, 是互联网版权产业智力资源配置合作行为 协调的核心要义。元 IP 创造者、版权运营平台合作 行为的协调, 可实现持续提高创意智力资源整体质量, 运营平台的满意度, 增加互联网版权产业价值链的总 效益。基于合理的收益分配，元 IP 创造者、版权营 运平台在产业价值链中通过合作共赢共同分享版权 
产业价值链的整体利益, 获取比单独行动更多的收益。

\section{3 互联网版权产业智力资源配置合作行为协 调的演化博弯模型}

\section{1 基本假定与参数假设}

依据互联网版权产业智力资源配置合作行为协 调的内容与影响因素, 设如下基本假定:

(1) 互联网版权产业的产业链由元 IP 创作者和 互联网版权运营商这两类群体组成。

(2) 元 IP 创作者和互联网版权运营商分别都有 两个可以选择的策略。元 IP 创作者可以有“合作”互 联网版权开发商 $\left(A_{1}\right)$ 和“不合作”开发平台而选择其 他方式筹得资金 $\left(A_{2}\right)$, 采取这两种策略的概率分别 为 $p$ 和 $1-\mathrm{p}(0 \leq \mathrm{p} \leq 1)$ 。

(3) 当互联网版权开发商选择“合作”策略时, 元 IP 创作者和互联网版权运营商行为协调, 项目能 有较高的开发成功率 $\alpha(0<\alpha<1)$ 。与此相反, 若开发商 仅靠个人以往经验, 此时成功率较低, 设为 $\beta(0<\beta<\alpha<1)$ 。

（4）互联网版权开发商（版权营运平台）可选 择采取的策略集为 $\{$ 合作，不合作\}：“合作”是指向元 IP 创作者提供高效服务（ $\mathrm{B}_{1} ）$; “不合作”是指向元 IP 创作者提供低效服务 $\left(\mathrm{B}_{2}\right)$ ，选择“合作”或“不合 作”的概率分别为 $\mathrm{q}$ 和 $1-\mathrm{q}(0 \leq \mathrm{q} \leq 1)$ 。

（5）互联网版权产业价值链保持稳定状态的前 提是元 IP 创作者与版权开发商相协调; 其中一方或 双方选择“不合作”时, 互联网版权产业价值链则不能 保持稳定。

(6) 元 IP 创作者的开发版权项目所需总金额是 $\mathrm{Q}$ 。若拒绝合作平台, 元 IP 创作者则需要自寻找满
足期望的开发商或开发平台，但因信息的不对称性、 交易合作成本不确定等, 需要付出较大的搜索成本 $r Q(0<r<1)$ 。元 IP 创作者选择合作时，假设合作高质 量的平台, 所得收益为: $\alpha[(1-\lambda) Q+e Q]$; 假设合 作低质量平台, 所得收益为 $\beta(1-\lambda) Q$ 。若元 IP 创作 者选择不合作时, 所得收益为 $(1-r) Q$ 。

（7）互联网版权智力资源配置合作向元 IP 创作 者提供服务时产生固定成本, “合作”型互联网版权开 发商的成本为 $C_{1}$, 合作提高服务质量也产生相应的 机会成本 $C(C>0)$ 。“不合作”时平台的成本为 $C_{2}\left(0<C_{2}<C_{1}\right)$, 但是在版权价值发现机制与约束 机制尚需完善的大环境下, “不合作”平台同样产生机 会成本 $R(0<R<C)$; 当互联网版权开发商选择“合 作”策略时, 若元 IP 创作者选择合作, 平台收益为 $\alpha \lambda Q-C_{1}-C$, 若元 IP 创作者选择不合作, 则平 台收益为 $-C$ 。当互联网版权开发商选择提供低质 量服务, 若元 IP 创作者选择合作, 平台收益为 $\beta \lambda Q-C_{2}-R$, 若元 IP 创作者选择不合作, 平台收 益则为 $-R$ 。

\section{2 演化博竕支付矩阵}

为了简化模型的参数设计又不失一般性, 本研究 假设各符号均大于零。基于上述参数假设, 构建互联 网版权智力资源配置合作行为博弯的收益矩阵, 如表 1 所示。

表 1 互联网版权智力资源配置合作行为博亦的收益矩阵

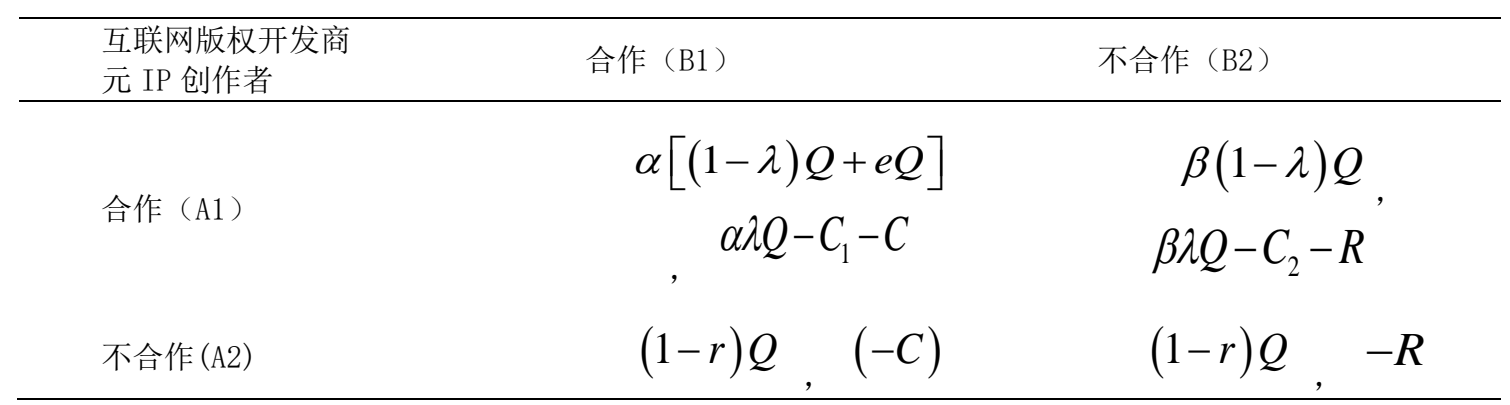

对表 1 中的收益做出如下假设:

(1) $\beta(1-\lambda) Q<(1-r) Q$, 低质量服务的 互联网版权开发商因其成功率较低, 元 IP 创作者对 于吸引受众注意力资源的需求难以达到预期, 而会进 行其他的行为选择。
(2) $\alpha \lambda Q-C_{1}>\beta \lambda Q-C_{2}$, 开发商合作收 益大于不合作收益。

\section{3 复制动态及其演化稳定策略}

首先, 根据表 1 的收益矩阵, 元 IP 创作者选择“合 作”平台时，其期望收益为: 


$$
\begin{aligned}
& U_{1}=q \alpha[(1-\lambda) Q+e Q]+(1-q) \beta(1-\lambda) Q \\
& =q[(1-\lambda)(\alpha-\beta)+\alpha e] Q+(1-\lambda) \beta Q \\
& -[\alpha(1-\lambda+e) Q-(1-r) Q(R-C)]
\end{aligned}
$$
益为:

当元 IP 创作者选择“不合作”平台时, 其期望收

$$
U_{2}=q[(1-r) Q]+(1-q)[(1-r) Q]=(1-r) Q
$$

元 IP 创作者群体的平均期望收益为:

$$
\bar{U}=p U_{1}+(1-p) U_{2}
$$

元 IP 创作者的复制动态方程为:

$$
F(p)=\frac{d p}{d t}=p\left(U_{1}-\bar{U}\right)=p(1-p)\left(U_{1}-U_{2}\right)
$$

对公式 (4) 进行求导,

$$
F^{\prime}(p)=\left(\frac{d p}{d t}\right)^{\prime}=(1-2 p)\left(U_{1}-U_{2}\right)
$$

令 $F(p)=0$, 则 $p=0, p=1$ 或 者 $\mathrm{q}^{*}=\frac{(1-r)-(1-\lambda) \beta}{(1-\lambda)(\alpha-\beta)+\alpha e}$

当 $\mathrm{q}=\mathrm{q} *$ 时, $F^{\prime}(p) \equiv 0$, 此时 $\mathrm{p}$ 可取所有点做 稳定点。当 $q \neq q *$ 时, $p=0$ 和 1 为稳定状态点。

当 $\mathrm{q}<\mathrm{q}$ *时, 求解得到的 $F^{\prime}(0)<0, F^{\prime}(1)>0$, $\mathrm{p}=0$ (不合作) 为稳定状态点。当 $\mathrm{q}>\mathrm{q}$ *时, 求解得到 $F^{\prime}(0)>0, F^{\prime}(1)<0, \mathrm{p}=1$ (合作) 为稳定状态 点。

进一步, 根据表 1 的收益矩阵, 当互联网版权开 发商选择“合作”时，其期望收益为:

$$
V_{1}=p\left(\alpha \lambda Q-C_{1}-C\right)+(1-p)(-C)
$$

对于不合作策略对应的期望收益为:

$$
V_{2}=p\left(\beta \lambda Q-C_{2}-R\right)+(1-p)(-R)
$$

平均期望收益为:

$$
\bar{V}=q V_{1}+(1-q) V_{2}
$$

互联网版权开发商的复制动态方程为:

$$
F(q)=\frac{d q}{d t}=q\left(V_{1}-\bar{V}\right)=q(1-q)\left(V_{1}-V_{2}\right)
$$

对 (9) 进行求导,

$$
F^{\prime}(q)=\left(\frac{d q}{d t}\right)^{\prime}=(1-2 q)\left(V_{1}-V_{2}\right)
$$

令 $\mathrm{F}(\mathrm{q})=0$, 则 $\mathrm{q}=0, \mathrm{q}=1$ 或 者 $\mathrm{p}^{*}=\frac{C-R}{(\alpha-\beta) \lambda Q-C_{1}+C_{2}}$ 。

当 $\mathrm{p}=\mathrm{p}$ *时, 无论 $\mathrm{q}$ 如何取值, $F^{\prime}(\mathrm{q}) \equiv 0$,

此时 $\mathrm{q}$ 可取所有点做稳定点。当 $\mathrm{p} \neq \mathrm{p}$ *时, $\mathrm{q}=0$ 和 1 为稳定状态点。

当 $\mathrm{p}<\mathrm{p}$ *时, 求解得到的 $F^{\prime}(0)<0, F^{\prime}(1)>0$, $\mathrm{q}=0$ (不合作) 为稳定状态点。当 $\mathrm{p}>\mathrm{p}$ *时, 求解得到 $F^{\prime}(0)>0, F^{\prime}(1)<0, \mathrm{q}=1$ (合作) 为稳定状态点。

联立复制动态方程 4 和 9 构成方程组, 在平面 $\{(\mathrm{p}, q) 0 \leq p, q \leq 1\}$ 内可以得到 5 个均衡点 :

$$
(0,0),(0,1),(1,0),(1,1) \text {, }
$$

$$
\left(\mathrm{p}^{*}=\frac{C-R}{(\alpha-\beta) \lambda Q-C_{1}+C_{2}}, \mathrm{q}^{*}=\frac{(1-r)-(1-\lambda) \beta}{(1-\lambda)(\alpha-\beta)+\alpha e}\right),
$$

依据雅克比矩阵的稳定性判定方法可得到各均 衡点的稳定性（Friedman，1991）。分别对公式(4) 和(9)求关于 $p$ 和 $q$ 的偏导数, 可得系统的雅克比矩 阵为:

$$
\left.J\left[\begin{array}{l}
\frac{d F(p)}{d p} \frac{d F(p)}{d q} \\
\frac{d F(q)}{d p} \frac{d F(q)}{d q}
\end{array}\right]=\left[\begin{array}{l}
(1-2 p)\left\{\begin{array}{l}
q[(1-\lambda)(\alpha-\beta)+\alpha e] Q \\
+(1-\lambda) \beta Q-(1-r) Q
\end{array}\right. \\
q(1-q)\left[(\alpha-\beta) \lambda Q-C_{1}+C_{2}\right]
\end{array}\right\} \begin{array}{l}
p(1-p)[(1-\lambda)(\alpha-\beta)+\alpha e] Q \\
(1-2 q)\left\{p\left[(\alpha-\beta) \lambda Q-C_{1}+C_{2}\right]+R-C\right\}
\end{array}\right]
$$

行列式记为 Det J, 迹为 $\operatorname{Tr} \mathrm{J}$, 则: 


$$
\begin{aligned}
& \operatorname{DetJ}=(1-2 p)(1-2 q)\left\{\begin{array}{l}
q[(1-\lambda)(\alpha-\beta)+\alpha e] \\
+(1-\lambda) \beta Q-(1-r) Q
\end{array}\right\}\left\{\begin{array}{l}
p\left[(\alpha-\beta) \lambda Q-C_{1}+C_{2}\right] \\
+R-C
\end{array}\right\}- \\
& p(1-p)[(1-\lambda)(\alpha-\beta)+\alpha e] Q \cdot q(1-q)\left[(\alpha-\beta) \lambda Q-C_{1}+C_{2}\right] \\
& \operatorname{Tr} J=(1-2 p)\left\{\begin{array}{l}
q[(1-\lambda)(\alpha-\beta)+\alpha e] Q \\
+(1-\lambda) \beta Q-(1-r) Q
\end{array}\right\}+(1-2 q)\left\{\begin{array}{l}
p\left[(\alpha-\beta) \lambda Q-C_{1}+C_{2}\right] \\
+R-C
\end{array}\right\}
\end{aligned}
$$

\begin{tabular}{|c|c|c|c|c|c|}
\hline 均衡点 & DetJ & 符号 & $\operatorname{Tr} J$ & 符号 & 结果 \\
\hline $\begin{array}{l}p=0 \\
q=0\end{array}$ & {$[(1-\lambda) \beta Q-(1-r) Q](R-C)$} & + & $(1-\lambda) \beta Q-(1-r) Q+R-C$ & - & ESS \\
\hline $\begin{array}{l}p=0 \\
q=1\end{array}$ & $-[\alpha(1-\lambda+e) Q-(1-r) Q](R-C)$ & + & {$[\alpha(1-\lambda+e) Q-(1-r) Q]-(R-C)$} & + & $\begin{array}{c}\text { 不稳 } \\
\text { 定 }\end{array}$ \\
\hline$p=1$ & $-[(1-\lambda) \beta Q-(1-r) Q]$ & & $-[(1-\lambda) \beta Q-(1-r) Q]+$ & & 不稳 \\
\hline$q=0$ & {$\left[(\alpha-\beta) \lambda Q-C_{1}+C_{2}+R-C\right]$} & + & {$\left[(\alpha-\beta) \lambda Q-C_{1}+C_{2}+R-C\right]$} & + & 定 \\
\hline$p=1$ & {$[\alpha(1-\lambda+e) Q-(1-r) Q]$} & & $-[\alpha(1-\lambda+e) Q-(1-r) Q]-$ & & \\
\hline$q=1$ & {$\left[(\alpha-\beta) \lambda Q-C_{1}+C_{2}+R-C\right]$} & + & {$\left[(\alpha-\beta) \lambda Q-C_{1}+C_{2}+R-C\right]$} & - & ESS \\
\hline $\begin{array}{l}p=p^{*} \\
q=q^{*}\end{array}$ & $-\left(1-p^{*}\right)\left(1-q^{*}\right)(C-R)[(1-r)-(1-\lambda) \beta]$ & - & 0 & & 鞍点 \\
\hline
\end{tabular}

结果如表 2 所示:

表 2 互联网版权开发商与元 IP 创作者演化博亦的稳定性分析

在复制动态方程的基础上, 对演化过程进行描述, 图如图 1 所示。

并得到五个均衡点, 对稳定性分析, 模型的演化相位

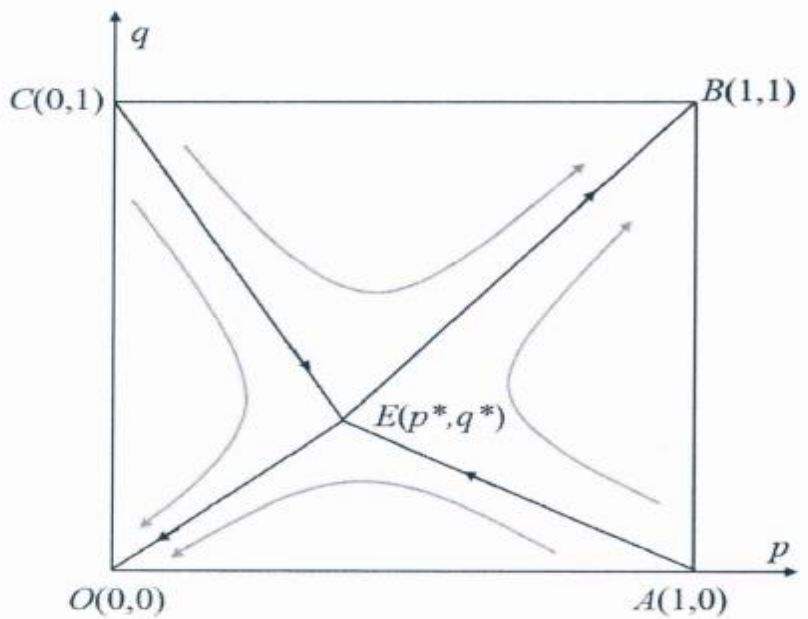

图 1 元 IP 创作者与互联网版权开发商行为协调的演化相位图

图 1 描述了互联网版权产业价值链元 IP 创作者 与互联网版权开发商行为协调的动态演化过程。点 $\mathrm{O}(0,0)$ 和点 $\mathrm{B}(1,1)$ 是演化均衡稳定点, 分别代表 IP
创作者与互联网版权开发商采取 (不合作，不合作) 和（合作，合作）的策略组合。点 $\mathrm{A}(1,0)$ 和点 $\mathrm{C}$ $(0,1)$ 为不稳定点, 点 $E\left(p^{*}, q^{*}\right)$ 为鞍点。在 
AEBC 区域里, 系统收敛于点 B $(1,1)$ 。此时元 IP 创作者与互联网版权开发商间形成完全协调状态; 在 AEBO 区域，系统收玫于点 $O$, 此时元 IP 创作者与 互联网版权开发商间形成完全不协调状态。

\section{4 演化博弃的数值仿真}

互联网版权开发商与元 IP 创作者之间的博弯系 统的作用机制包括有利因子和不利因子。其中有利因 子包括元 IP 创作者的注意力资源吸引程度 $\mathrm{Q}$ 、互联 网版权开发商收取的服务费率、互联网版权开发商提 供高服务的效率（ $a$ 和 $\mathrm{e}$ ）以及政府监管 $(\mathrm{R})$ ；不 利因子主要是互联网版权开发商提供高服务付出的 成本。

为了更好验证博弯的正确性并给出各参数的影
响，本研究运用 Matlab 软件进行数值仿真。用到的 参数取值如下: $\mathrm{Q}=300, a=0.8, \mathrm{e}=0.5, \lambda=0.1$, $\mathrm{C}=7, \mathrm{R}=3, \quad \mathrm{C}_{1}=5, \mathrm{C}_{2}=1, \mathrm{r}=0.4$ 。

\section{1 注意力资源吸引程度对系统演化的影响}

保持其他参数不变, 令初始值 $(p, q)=(0.2,0.9)$, 当 $Q=200 、 800 、 1000 、 2500$ 时，系统演化的路径 如图 2 所示。从图可知, 元 IP 创作者的内容吸引受 众注意力资源的需求达到一定值时, 系统才会收玫于

（合作，合作）的平衡点，且吸引受众注意力资源的 需求越大，收敛速度越快。可见元 IP 创作者的注意 力资源吸引程度是互联网版权开发商运行的基础, 对 互联网版权开发商合作开展高质量的 IP 开发有正向 的激励作用。

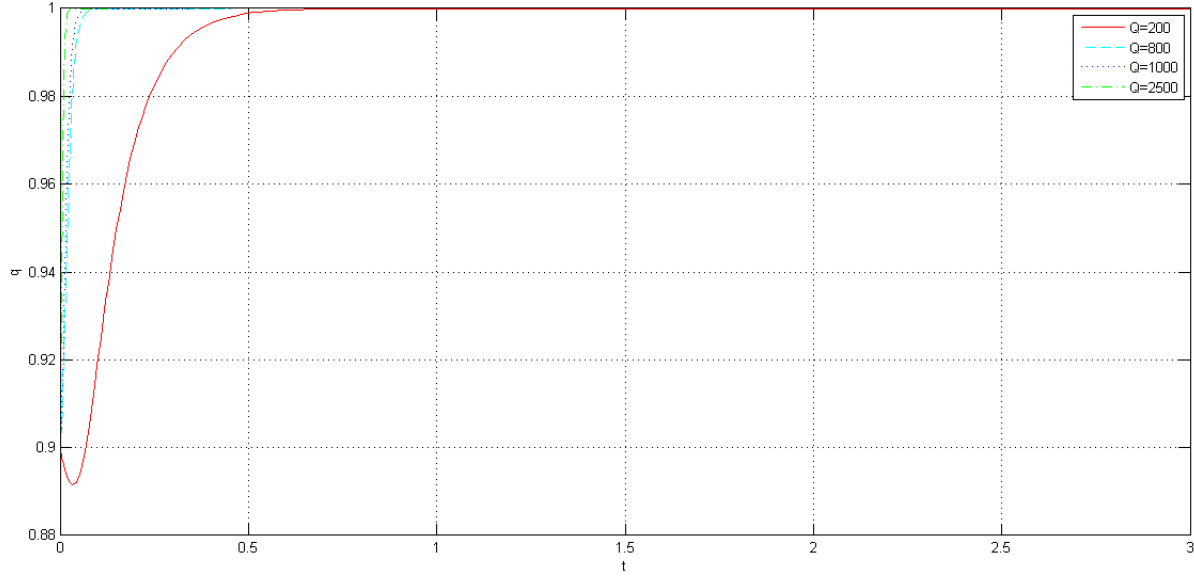

图 2 不同 $\mathrm{Q}$ 值下 $\mathrm{q}$ 的演化路径

\section{2 参与主体利益分配率对系统演化的影响}

为了考察参与主体利益分配率对系统演化的影 响, 在模型中保持其他参数不变, 令初始值 $(p, q)=$ $(0.2,0.9), \lambda=0.01,0.3,0.5$ 时 $\mathrm{q}$ 的路径图如图 3。 由图 3 可知, 提高利益分配率对互联网版权开发商采 取“合作”策略有正向激励作用。对于元 IP 创作者,
提高利益分配率最初会导致元 IP 创作者参与互联网 版权产业创意智力资源配置成本增加而采取“不合作” 策略，但随着互联网版权开发商积极选择“合作”策略， 元 IP 创作者参与互联网版权产业创意智力资源配置 收益提高从而转向“合作”策略。可见，在博弯的演化 过程中, 版权开发商的行为选择会对双方后续的合作 产生重要的影响。

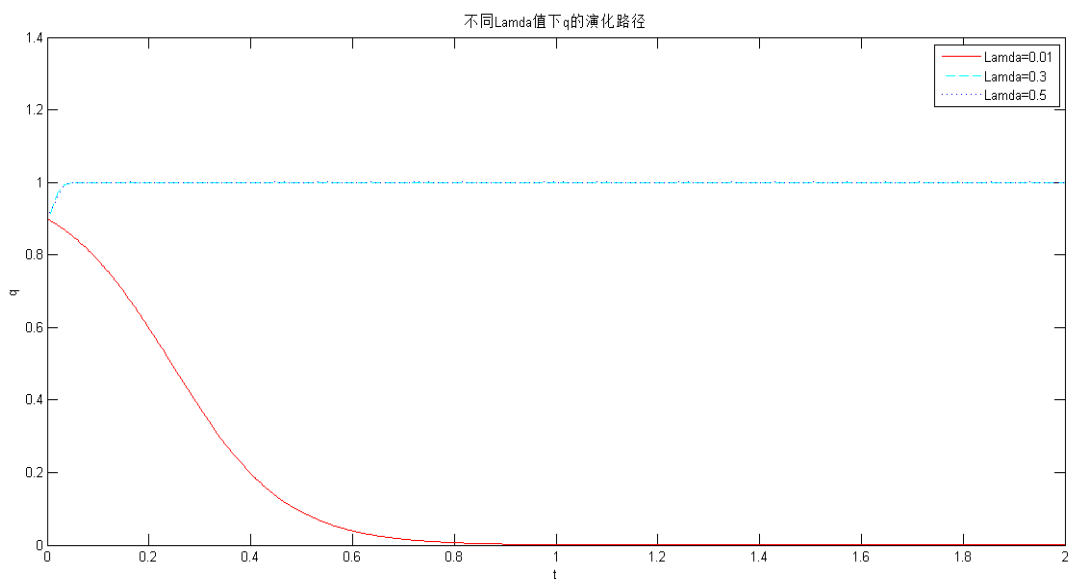

图 3 不同 $\lambda$ 值下 $\mathrm{q}$ 的演化路径 


\section{3 创意智力资源开发效用对系统演化的影 响}

为了考察提高创意智力资源的开发效用对系统 演化的影响, 在模型中观察 $\alpha$ 的变化, 此处 $\alpha$ 表示当 互联网版权开发商选择“合作”策略时, 版权内容开发
成功率。保持其他参数不变, 令初始值 $(p, q)=(0.2$, $0.9), \alpha=0.6 、 0.65 、 0.7 、 0.75$ 时 $\mathrm{q}$ 的路径图如图 4。 结果显示, 系统收敛速度与项目成功率 $\alpha$ 正相关, 且 为了保证系统向合作方向演化， $\alpha$ 需足够大。可见， 通过提高创意智力资源的开发效用, 提高版权内容开 发成功率, 能够促使价值链的参与主体形成合作关系。

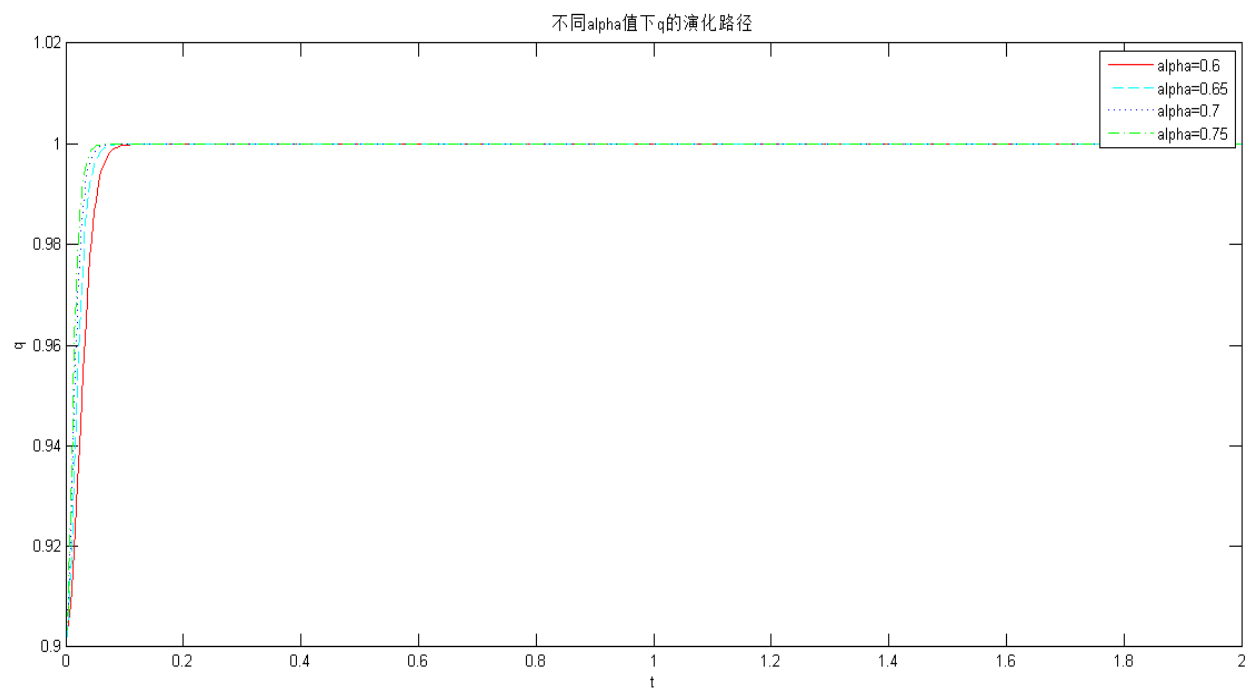

图 4 不同 $\alpha$ 值下 $\mathrm{q}$ 的演化路径

接下来分析 e 对系统演化的影响 (e 表示若合作 互联网版权开发商及互联网版权开发商选择“合作” 策略, 由于成员间合作行为的协调, 元 IP 创作者能 得到额外的社会效益系数)。保持其他参数不变, 令
初始值 $(p, q)=(0.7,04), e=0.4,0.6,0.8,1.0$ 时 $\mathrm{q}$ 的路径图如图 5。可以看出, 互联网版权开发商合 作提高服务质量对系统向（合作，合作）演化有正反 馈作用，对收敛效果却不明显。

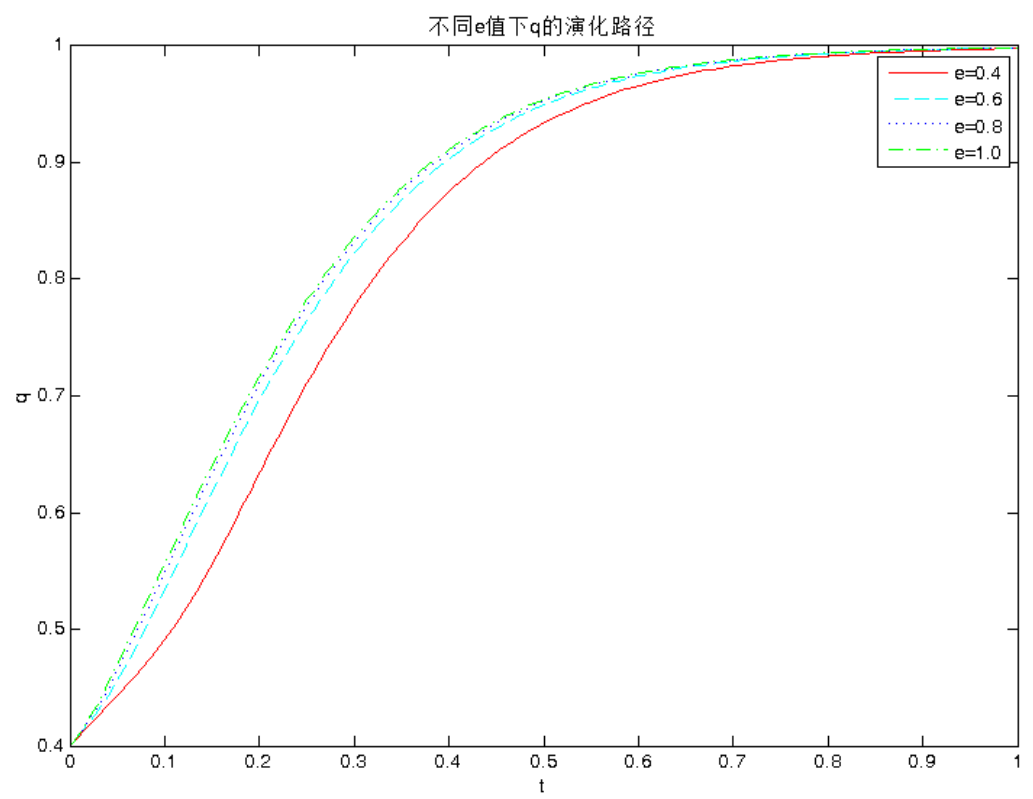

图 5 不同 $\mathrm{e}$ 值下 $\mathrm{q}$ 的演化路径

由图 4 和图 5 可知, 互联网版权开发商合作降低 与元 IP 创作者之间的信息不对称, 提高 IP 开发成功 率比为元 IP 的创作者创造额外社会效益更能推动系 统向（合作，合作）均衡演化。

\section{4 合作开发成本对系统演化的影响}

单独考虑合作开发成本的影响。令初始值 $(\mathrm{p}, \mathrm{q})$ $=(0.2,0.9), \quad \mathrm{C}=15 、 25 、 35$ 时 $\mathrm{q}$ 的路径图如图 6。 
这里的 C 表示互联网版权智力资源配置合作与元 IP 创作者合作提高服务质量面临的机会成本。由图 6 可知, 成本与系统向合作演化反相关。这代表机会成
本的提高将导致互联网版权开发商在合作开展高质 量的 IP 开发时遭遇更多的风险和代价等。

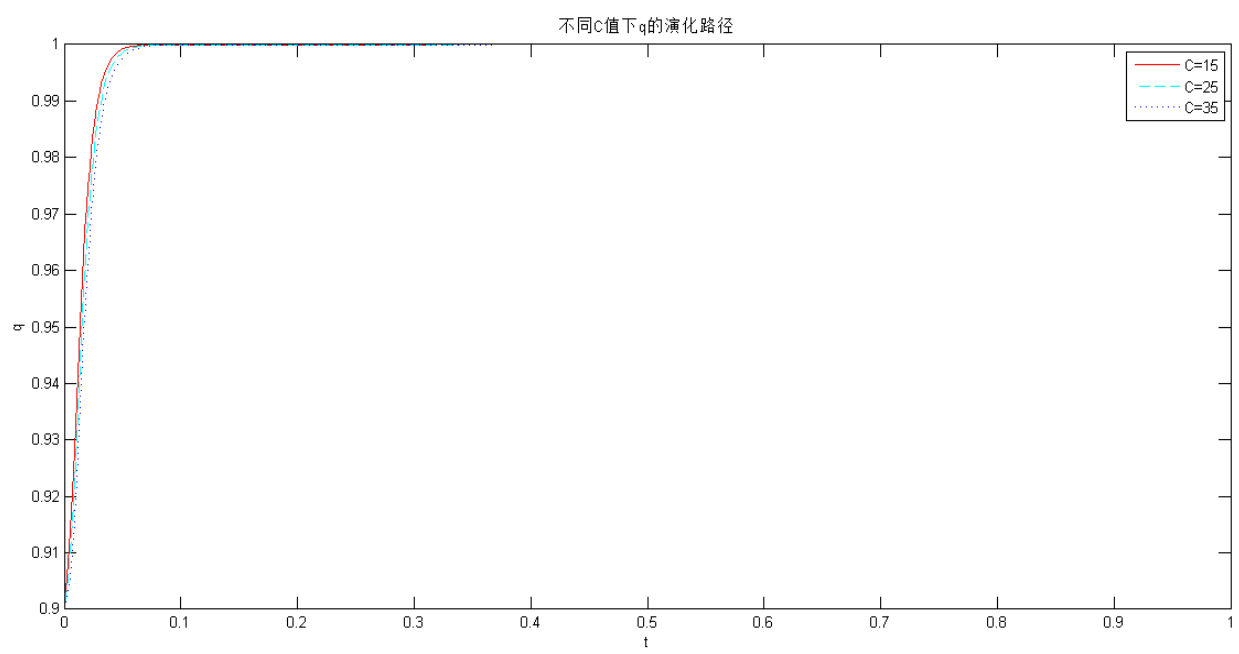

图 6 不同 $\mathrm{C}$ 值下 $\mathrm{q}$ 的演化路径

\section{5 开发商的机会成本对系统演化的影响}

考虑开发商的机会成本的影响。令初始值 $(p, q)$ $=(0.2,0.9), \mathrm{R}=2,3,4,5$ 时 $\mathrm{q}$ 的路径图如图 7。 这里的 $\mathrm{R}$ 表示在版权价值发现机制与约束机制尚需
完善的大环境下, “不合作”平台面临的机会成本 $(0<R<C)$ 。高质量的互联网版权开发商的机会成本 能推动系统向 (合作, 合作) 策略演化。政府监管 和设置准入标准为主要影响因素, 对互联网版权产业 创意智力资源配置的引导和监控有重要影响。

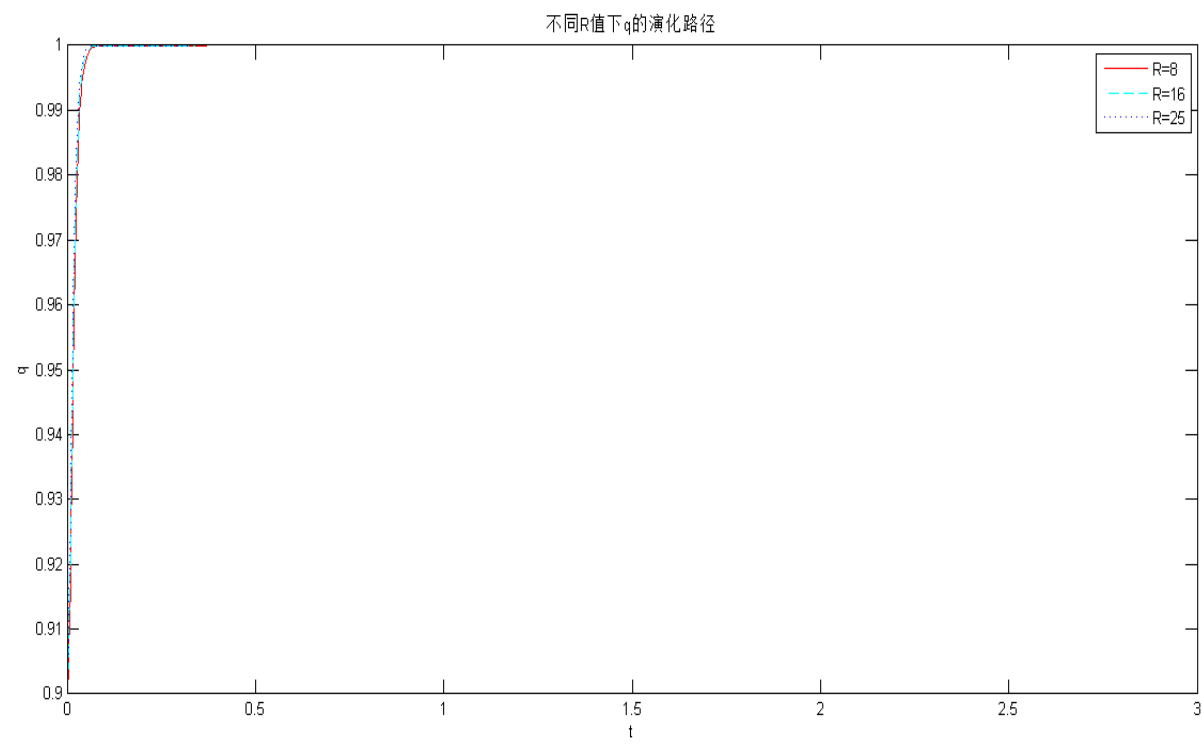

图 7 不同 $\mathrm{R}$ 值下 $\mathrm{q}$ 的演化路径

在博弯模型中，互联网版权开发商与元 IP 创作 者之间的博亦系统有两个均衡点, 一个均衡点是互联 网版权开发商选择合作提供高质量服务, 元 IP 创作 者吸引足够的注意力资源, 而另一个均衡点是互联网 版权开发商不合作并只提供低水平的服务, 元 IP 创 作者无法获得足够的注意力资源。显然, 在系统的博 亦推演过程中, 我们更期待的是第一个均衡点的出现, 避免第二种情况发生。系统最终演化方向由创意智力
资源配置的市场环境和开始双方选择比例决定。

\section{5 结论及启示}

（1）通过对元 IP 创作者利益分配比例进行优化, 在价值链中提升创作者的参与积极性。互联网版权产 业创意智力资源配置可以通过优化元 IP 创作者利益 分配比例, 在版权产业价值链中提升创作者的参与积 
极性，进而产出更多的优质元 IP，为版权开发机构 提供二次开发新机会。

(2) 提升互联网版权开发商对元 IP 内容的开发 效率。通过提高创意智力资源的开发效用, 提高 IP 开发成功率, 能够促使价值链的参与主体形成合作关 系。在此过程中, 有必要引入互联网思维, 特别是其 对共享的要义。在传统媒介环境下, 出版机构对元 IP 的改编、重组效率相对较低, 互联网思维下的文化产 业运营各环节中的产品关联性和共生性强, 以动态高 效的产业链为依托, 完成各相关产业板块的高效开发, 打造新型产业价值链, 满足人民全方位消费需求。

(3) 互联网版权开发商运营成本应适当控制。 一个主要原则就是将成本用在最需要的地方, 同时需 要控制投入的风险。在版权开发过程中, 版权开发商 应结合自身的主营业务有选择的将一些非核心业务 外包，从而更有针对性地控制成本、提升发展。

(4) 行业监管标准应进一步健全。中国目前的 互联网版权产业发展仍处于自由生长阶段, 版权开发 盲目、文娱项目过度开发的现象明显, 互联网版权开 发商的服务能力各有差别。政府监管和设置准入标准 为主要影响因素, 对互联网版权产业创意智力资源配 置的引导和监控有重要影响。需要进一步规范行业发 展、优化产业规制机制。

（5）互联网版权产业价值链发展需要各环节相 互合作共同实现价值链的增值。鼓励更多的专业性企 业参与到价值开发环节, 积极打造 IP 品牌, 利用品 牌效应延伸产业链, 共同实现价值增值和优势地位。 这对利益分配模式和资费模式提出了新的要求。所以, 要充分调动价值链上下游主体作用 ${ }^{[10]}$, 通过合理的 收益分成鼓励更多的价值链成员更好朝着良性方向 发展。

\section{6 结束语}

本文提出了互联网版权产业价值链智力资源配 置行为协调的概念, 应用演化博弯理论, 通过模型的 构建及求解分析, 研究了博弯演化过程及影响因素, 为互联网版权产业发展提供了有益借鉴。结果显示, 协调演化方向受协调成本、成本分推比例、单独合作 收益等因素的影响。因此在收益分配机制和管理模式 等方面的优化, 可以进一步推动互联网版权开发行为 向全面协调的方向演化。

\section{REFERENCES}

[1]Ernan Haruvy, Ashutosh Prasad.Optimal Freeware Quality in the Presence of Network Externalities: an Evolutionary Game Theoretical Approach[J],Journal of Evolutionary Economics,2001(11):231—248

[2]Poter M.E. Competitive Advantage:Creating and Sustaining Superior Performance.London:The free Press, 1985 ,

[3]Stevevs, Graham.Integeating the Supplying chain[J],International Journal of Physical Distribution and Material Management,1989,19(8):3-8

[4] Wang H , Mu R, Xie J . Study on Market Competition Behaviors of Mobile Communication Industry in China Based on the Game Theory[C]// International Conference on Mechatronics. 2016.

[5]Risvan Dirza, Sigurd Skogestad, Dinesh Krishnamoorthy. Optimal Resource Allocation using Distributed Feedback-based Real-time Optimization [J], IFAC-PapersOnLine, 2021, 54(3):706-711

[6]Wenliu Cai, Hongxia Wu. Research on the optimal allocation of intellectual resources based on data mining analysis $[\mathrm{J}]$, Boletin Tecnico/Technical Bulletin, 2017, 55(10):225-232

[7]Peilian Guo, Changda Han. Nash equilibrium and group strategy consensus of networked evolutionary game with coupled social groups $[\mathrm{J}]$, Applied Mathematics and Computation, 2021, 409:126380

[8]Jing S , Ren L . A Study on the Benefit Distribution of Mobile Publishing Industrial Chain Based on the Cooperative Game Theory[J]. Springer Berlin Heidelberg, 2012.

[9] Yang W B , Gao Z L . Non-empty Property of the Core in Cooperative Games of the Supply Chain Value Network[J]. Systems Engineering, 2009, 27(11):77-81.

[10]Kenneth S.Corts. The Strategic Effects of Vertical Market Structure:Common Agency and Divisionalization in the US Motion Picture Industry[J].Journal of Econmics \& Management Strategy,2001,10(4):513 